\title{
A Brief Narrative Summary of Randomized Controlled Trials Investigating EMDR Treatment of Patients With Depression
}

\author{
Francesca Malandrone \\ Sara Carletto (1) \\ University of Turin, Turin, Italy \\ Michael Hase \\ Lüneburg Center for Stress Medicine, Lüneburg, Germany \\ Arne Hofmann \\ EMDR Institut Deutschland, Bergisch Gladbach, Germany \\ Luca Ostacoli \\ University of Turin, Turin, Italy
}

\begin{abstract}
Depression, one of the most common mental disorders, is characterized by enormous social costs and limited rates of treatment success, even though psychotherapeutic and pharmacological treatments currently contribute to an increase in the remission rate. In light of recent studies that have shown that traumas and adverse life experiences may represent risk factors for the onset of depression, the therapeutic approach of eye movement desensitization and reprocessing (EMDR) therapy has been seen as potentially effective in the treatment of depression. The purpose of the present brief narrative review is to summarize the current literature on the efficacy of EMDR in patients with depression, in particular by referring to randomized controlled clinical trials (RCTs) that examined depression as a primary outcome. The data examined are updated to March 2019 and count seven RCT studies covering the years from 2001 to 2019. They are heterogeneous by type of intervention and demographic characteristics of the sample. Although the selected studies are few and with different methodological critical issues, the findings reported by the different authors suggest in a preliminary way that EMDR can be a useful treatment for depression.
\end{abstract}

Keywords: eye movement desensitization and reprocessing; EMDR; depression; review; randomized controlled trial

D epression is a common mental disorder and one of the leading causes of disability worldwide, with huge individual and societal costs (World Health Organization, 2017). Given the tremendous disease burden, different treatment options for this disorder are available. They range from lowintensity psychosocial interventions to high-intensity psychological interventions integrated with pharmacotherapy, depending on symptom severity (National Institute for Health and Care Excellence, 2018).

Among psychological interventions, cognitive behavioral therapy (CBT), interpersonal psychotherapy, problem-solving therapy, behavioral activation, and short-term psychodynamic therapy have been found to be equally effective in treating depressive disorders (Barth, Michlig, \& Munder, 2014; Cuijpers, Van Straten, Andersson, \& Van Oppen, 2008). Nevertheless, the proportion of patients who recover after treatment is still limited, with relapse rates estimated around $50 \%$ after 2 years (Hollon et al., 2002; Vittengl, 
Clark, Dunn, \& Jarrett, 2007). Therefore, it is essential to identify how to improve treatment outcomes.

In recent years an important correlation between exposure to adverse experiences during childhood and the development of depression in adulthood has been highlighted (Li, D’Arcy, \& Meng, 2016; Nemeroff, 2016; Norman et al., 2012), suggesting that traumafocused treatments may contribute to the treatment of this disorder. Eye movement desensitization and reprocessing (EMDR) is an evidence-based therapy for the treatment of psychological trauma sequelae and could represent a valid therapeutic option for the treatment of patients suffering from depression, either as a stand-alone treatment or as an add-on to other interventions. The first report of patients with depressive disorder treated with EMDR was described in the late 1990s (Manfield, 1998). In the following years, several other case and uncontrolled studies were published. The results of these studies have been summarized by two recent reviews (Carletto et al., 2017; Wood \& Ricketts, 2013). In their review, Wood and Ricketts (2013) described the historical development of clinical and research applications of EMDR therapy for the treatment of depression as a primary diagnosis, reporting the results of the first case and uncontrolled studies; in the second review, Carletto et al. (2017) summarized the results of only controlled studies, also adding an evaluation of the study quality. Both reviews concluded that EMDR should be considered a promising approach, even though they highlighted several limitations that were mainly related to the small number of randomized controlled studies at that time. In recent years the interest of researchers and clinicians has continued to grow and several other controlled and randomized studies have been conducted.

The aim of this brief narrative review is to provide a summary presenting the results of the randomized controlled studies currently available in the literature on this topic. Literature search was carried out by searching "EMDR" or "Eye Movement Desensitization and Reprocessing," and "depress ${ }^{\star}$ " as keywords in principal databases (e.g., PubMed, PsychINFO) and in the Francine Shapiro Library; studies with a randomized controlled trial (RCT) design which investigated depression as primary outcome were included with no limitations related to type of population, age, comorbidity with organic disease, or pharmacotherapy.

To the best of our knowledge, seven RCTs have been conducted for the evaluation of the efficacy of EMDR therapy in treating depressive symptoms as a primary diagnosis. Characteristics of those studies are shown in Table 1. All studies included adult patients.
Studies had samples composed from 22 to 82 participants who had a depression diagnosis only in six studies, whereas in one study patients had a concurrent general medical condition. Five studies specified the concomitant use of antidepressant therapies, while in the others it was not specified or it was an exclusion criterion. The number of sessions of EMDR treatment ranged from 3 to 24 . A variety of measures were applied to evaluate depressive symptoms: four studies used the Beck Depression Inventory (BDI), one study employed the Hamilton Depression Scale, one study used both the Symptom Checklist-90 (SCL-90) and the BDI-II, and one study used both the MontgomeryAsberg Depression Rating Scale (MADRS) and the BDI-II.

\section{Summary of Findings}

In two studies EMDR was compared with no treatment, that is, waiting lists or no-intervention. Behnam Moghadam (2015) conducted a study involving patients with myocardial infarction to evaluate EMDR in treating depression. The control group received no intervention. The experimental group reported, compared to the control group, a significant reduction in depressive symptoms with depressive symptomatology levels below the clinical threshold following treatment. Mauna Gauhar (2016) administered EMDR to patients with major depression who showed a significant improvement in symptoms compared to the control group (waiting list). This improvement was also identified at the 3-month follow-ups.

Three studies evaluated EMDR in comparison with another active intervention. In his unpublished dissertation, Hogan (2001) investigated the efficacy of a single session of EMDR or CBT. Although no significant differences were observed between the two groups, which proved to be equally effective in treating depressive symptoms, it is possible to differentiate them by analyzing the data of individual participants. In fact, four people belonging to the EMDR group show an almost complete remission of depressive symptoms. In comparison, it was not possible to observe such a symptom reduction in the CBT group. In addition, patients reported that EMDR treatment was less negative than CBT "primarily due to the increased awareness of negative thoughts common to CBT but not experienced in EMDR treatment" (Hogan, 2001). Ostacoli et al. (2018) published the first RCT comparing EMDR with CBT in patients with recurrent depressive episodes and reported that both interventions were effective in reducing clinical levels of depression, although a statistically significant 
increase in EMDR treatment was observed at the end of the intervention phase. Minelli et al. (2019) compared EMDR with trauma-focused cognitive behavioral therapy (TF-CBT) in patients with treatment resistant depression treated with different types of psychosocial and/or pharmacological therapies. EMDR was shown to be as effective as TF-CBT in reducing depression symptoms during hospitalization; however, at the follow-up visit, only EMDR maintained this amelioration.

Finally, EMDR was evaluated as an adjunctive treatment in two studies. In the research conducted by Lei and Zhen-Ying (2007), an intervention involving the addition of EMDR sessions to therapy with sertraline was compared with the administration of sertraline alone. Although the data collected showed no statistically significant differences between the two groups, the combination of EMDR with sertraline appears to have produced a more rapid effect, contributing to greater safety and better compliance with therapies. Hase et al. (2018) compared the efficacy of EMDR combined with psychoeducation to psychoeducation alone in patients with depression, showing a significant improvement in those patients who received adjunctive EMDR.

As regards EMDR protocols used to treat patients, four studies (Behnam Moghadam, 2015; Hogan, 2001;
Lei \& Zhen-Ying, 2007; Minelli et al., 2019) referred to the standard EMDR protocol of Shapiro (1995; Shapiro, 2001). One study (Mauna Gauhar, 2016) used the Shapiro's standard protocol but with a slight modification in Phase 3 during target assessment: patients were asked to first identify the negative cognition most strongly associated with reduced functions and then to identify the related disturbing event. In the other two studies (Hase et al., 2018; Ostacoli et al., 2018) the EMDR treatment followed the DeprEND protocol: that is, the manual for EMDR in the treatment of depressive patients (see Hofmann et al., 2016 for a detailed explanation).

\section{Discussion}

Most research on the application of EMDR for treating depressive disorders carried out in the last 18 years investigated the use of the Shapiro standard protocol (Shapiro, 2001). In 2016 the DeprEND protocol was published to define a specific protocol for this kind of population, thus addressing different types of memories such as traumatic memories or depressive and suicidal states (Hofmann et al., 2016).

RCTs that have evaluated EMDR efficacy on the improvement of depressive symptoms are few and

TABLE 1. Characteristics of Seven Randomized Controlled Clinical Trials

\begin{tabular}{|c|c|c|c|c|}
\hline Author (Year) & $\mathrm{N}$ & Treatments/WL & $\begin{array}{l}\text { Number of } \\
\text { Sessions }\end{array}$ & Findings \\
\hline Hogan (2001) & 30 & EMDR versus CBT & 4 & $\mathrm{EMDR}=\mathrm{CBT}$ \\
\hline $\begin{array}{l}\text { Lei and Zhen-Ying } \\
\text { (2007) }\end{array}$ & 64 & $\begin{array}{l}\text { Sertraline + EMDR } \\
\text { versus Sertraline }\end{array}$ & 6 & $\begin{array}{l}\text { Sertraline }+ \text { EMDR }= \\
\text { Sertraline }\end{array}$ \\
\hline $\begin{array}{l}\text { Behnam Moghadam } \\
(2015)\end{array}$ & 60 & $\begin{array}{l}\text { EMDR versus TAU (no } \\
\text { intervention) }\end{array}$ & 3 (45-90 minutes) & $\mathrm{EMDR}>\mathrm{TAU}$ \\
\hline Mauna Gauhar (2016) & 26 & EMDR versus WL & 6 to 8 ( 1 hour) & EMDR > WL \\
\hline Ostacoli et al. (2018) & 82 & EMDR versus CBT & $15 \pm 3$ & $\begin{array}{l}\text { Posttreatment: } \\
\text { EMDR > CBT; } \\
\text { Follow-up: EMDR = CBT }\end{array}$ \\
\hline Hase et al. ( 2018) & 30 & $\begin{array}{l}\text { EMDR + TAU versus } \\
\text { TAU }\end{array}$ & $\begin{array}{l}1 \text { or } 2 \text { EMDR } \\
\text { sessions/week. } \\
\text { Minimum } 4 \\
\text { sessions, } \\
\text { maximum } 12 .\end{array}$ & $\mathrm{EMDR}+\mathrm{TAU}>\mathrm{TAU}$ \\
\hline Minelli et al. (2019) & 22 & EMDR versus TF-CBT & $\begin{array}{l}24 \text { (three } 1 \text {-hour } \\
\text { sessions/week, for } \\
8 \text { weeks) }\end{array}$ & $\begin{array}{l}\text { Posttreatment: EMDR = } \\
\text { TF-CBT; Follow-up: EMDR } \\
\text { > TF-CBT }\end{array}$ \\
\hline
\end{tabular}

Note. CBT = cognitive behavioral therapy; TF-CBT $=$ trauma-focused cognitive behavioral therapy; $\mathrm{WL}=$ waiting list; TAU $=$ treatment as usual.

$>$ indicates significantly better than. 
with some methodological concerns. However, the findings of these studies suggest that EMDR could be a compelling option for the depression treatment. In particular, greater evidence of effectiveness in the treatment of depressive symptoms was found when EMDR was compared with inactive control groups (e.g. waiting list). When EMDR was administered as an add-on to other treatments (e.g. sertraline or psychoeducation), authors reported the potentiality of boosting and strengthening the effects of the standard treatments.

Lastly, a direct comparison with CBT (Ostacoli et al., 2018) found that EMDR seemed to be comparable or slightly superior to $\mathrm{CBT}$, which is considered the gold standard intervention for the treatment of depression, according to clinical guidelines (National Collaborating Centre for Mental Health (UK), 2010). Moreover, results of the follow-up analysis suggest that EMDR improvements may have more potential to remain stable and maintained over time.

It is possible to identify some limitations concerning the studies in this field. Current evidence is heavily influenced by methodological flaws, including randomization details not reported and small sample size, and only two studies cited the DeprEND protocol. In fact, almost no information has been provided about treatment conceptualization nor the type of targets, making it difficult to compare the studies and to derive important clinical information. Therefore, findings are tentative and need to be supported by larger and more robust evaluations. Clinical interviews conducted by independent assessors must be used in the future. The main results available to date, in fact, are the result of self-reported measures, which have in themselves the risk of poor objectivity in measuring the effectiveness of the intervention (such as the potential overestimation). Only few studies included follow-up assessments, which are fundamental to evaluate longterm effects. Therefore, better designed research is needed to confirm the available findings.

The dissemination of the DeprEND protocol among clinicians and researchers is of the utmost importance, in order to obtain more comparable data and to increase the information about the effectiveness of this specific EMDR protocol. More broadly, beyond the methodological aspects, more information about treatment conceptualization, targets, and stabilization, as well as resources installation techniques should be provided. These recommendations have a repercussion at clinical and research levels, allowing to make the most of the potential of the EMDR treatment. Moreover, in order to expand the framework of knowledge on this topic, a systematic review and meta-analysis on the effects of EMDR treatment on depressive symptoms, both as primary and secondary outcome in controlled studies, is in progress by our research group (PROSPERO Register for Systematic Reviews registration number: CRD42018090086). In conclusion, even though more research is needed, EMDR could be considered a useful therapy to treat depression, as an alternative to gold standard treatments or in addition to routine clinical tools.

\section{References}

Barth, J., Michlig, N., \& Munder, T. (2014). Unique and shared techniques in cognitive-behavioural and short-term psychodynamic psychotherapy: A content analysis of randomised trials on depression. Health Psychology and Behavioural Medicine, 2(1), 929-950. doi:10.1080/21642850.2014.931231

Behnam Moghadam, M. (2015). Effect of eye movement desensitization and reprocessing (EMDR) on depression in patients with myocardial infarction. Global Journal Health Science, 7(6), 258-262. doi:10.5539/ Gjhs.V7n6p258

Carletto, S., Ostacoli, L., Colombi, N., Calorio, L., Oliva, F., Fernandez, I., \& Hofmann, A. (2017). EMDR for depression: A systematic review of controlled studies. Clinical Neuropsychiatry: Journal of Treatment Evaluation, 14(5), 306-312. doi:10.3389/fpsyg.2018.00534

Cuijpers, P., Van Straten, A., Andersson, G., \& Van Oppen, P. (2008). Psychotherapy for depression in adults: A meta-analysis of comparative outcome studies. Journal of Consulting and Clinical Psychology, 76(6), 909-922. doi:10.1037/a0013075

Hase, M., Plagge, J., Hase, A., Braas, R., Ostacoli, L., Hofmann, A., \& Huchzermeier, C. (2018). Eye movement desensitization and reprocessing versus treatment as usual in the treatment of depression: A randomized-controlled trial. Frontiers in Psychology, 9, 1384. doi:10.3389/fpsyg.2018.01384

Hofmann, A., Hase, M., Liebermann, P., Ostacoli, L., Lehnung, M., Ebner, F., . . . Tumani, V. (2016). DeprEnd(C-EMDR therapy protocol for the treatment of depressive disorders. In M. Luber \& M. Luber (Eds.), Eye movement desensitization and reprocessing (EMDR) therapy scripted protocols and summary sheets: Treating anxiety, obsessive-compulsive, and mood-related conditions (pp. 289311) New York, NY: Springer Publishing Co.

Hogan, W. A. (2001). The comparative effects of eye movement desensitization and reprocessing (EMDR) and cognitive behavioral therapy (CBT) in the treatment of depression. Dissertation Abstracts International: Section B: The Sciences and Engineering, 62(2-B), 1082. 
Hollon, S. D., Muñoz, R. F., Barlow, D. H., Beardslee, W. R., Bell, C. C., Bernal, G., . . . Sommers, D. (2002). Psychosocial intervention development for the prevention and treatment of depression: Promoting innovation and increasing access. Biological Psychiatry, 52(6), 610-630. doi:10.1016/S0006-3223(02)01384-7

Lei, S., \& Zhen-Ying, W. (2007). Comparative study of sertraline combined with EMDR in the treatment of depression. Journal of Clinical Psychosomatic, 4, 307-308.

Li, M., D’Arcy, C., \& Meng, X. (2016). Maltreatment in childhood substantially increases the risk of adult depression and anxiety in prospective cohort studies: Systematic review, meta-analysis, and proportional attributable fractions. Psychological Medicine, 46(4), 717730. doi:10.1017/S0033291715002743

Manfield, P. (1998). EMDR terms and procedures: Resolution of uncomplicated depression. In P. Manfield \& P. Manfield (Eds.), Extending EMDR: A casebook of innovative applications (pp. 15-36). New York, NY: W. W. Norton.

Mauna Gauhar, Y. W. (2016). The efficacy of EMDR in the treatment of depression. Journal of EMDR Practice and Research, 10(2), 59-69. doi:10.1891/1933-3196.10.2.59

Minelli, A., Zampieri, E., Sacco, C., Bazzanella, R., Mezzetti, N., Tessari, E., . . . Bortolomasi, M. (2019). Clinical efficacy of trauma-focused psychotherapies in treatment-resistant depression (TRD) in-patients: A randomized, controlled pilot-study. Psychiatry Research, 273, 567-574. doi:10.1016/j.psychres.2019.01.070

National Collaborating Centre for Mental Health (UK). (2010). Depression in adults with a chronic physical health problem: Treatment and management. Retrieved from http: / /www.ncbi.nlm.nih.gov/books/NBK82916/

National Institute for Health and Care Excellence. (2018). Depression in adults: Recognition and management. Retrieved from https://www.nice.org.uk/guidance $/ \operatorname{cg} 90$

Nemeroff, C. B. (2016). Paradise lost: The neurobiological and clinical consequences of child abuse and neglect. Neuron, 89(5), 892-909. doi:10.1016/j.neuron. 2016.01.019

Norman, R. E., Byambaa, M., De, R., Butchart, A., Scott, J., \& Vos, T. (2012). The long-term health consequences of child physical abuse, emotional abuse, and neglect: A systematic review and meta-analysis. PLOS Medicine, 9(11), e1001349. doi:10.1371/journal.pmed.1001349

Ostacoli, L., Carletto, S., Cavallo, M., Baldomir-Gago, P., Di Lorenzo, G., Fernandez, I., . . . Hofmann, A.
(2018). Comparison of eye movement desensitization reprocessing and cognitive behavioral therapy as adjunctive treatments for recurrent depression: The European Depression EMDR Network (EDEN) randomized controlled trial. Frontiers in Psychology, 9, 74. doi:10.3389/fpsyg.2018.00074

Shapiro, F. (1995). Eye movement desensitization and reprocessing: Basic principles, protocols and procedures (1st ed.). New York, NY: Guilford Press.

Shapiro, F. (2001). Trauma and adaptive informationprocessing: EMDR's dynamic and behavioral interface. In M. F. Solomon, R. J. Neborsky, \& L. McCullough (Eds.), Short-term therapy for long-term change (pp. 112129). New York, NY: W Norton \& Co.

Vittengl, J. R., Clark, L. A., Dunn, T. W., \& Jarrett, R. B. (2007). Reducing relapse and recurrence in unipolar depression: A comparative meta-analysis of cognitivebehavioral therapy's effects. Journal of Consulting and Clinical Psychology, 75(3), 475-488. doi:10.1037/0022006X.75.3.475

Wood, E., \& Ricketts, T. (2013). Is EMDR an evidencedbased treatment for depression? A review of the literature. Journal of EMDR Practice and Research, 7(4), 225-235. doi:10.1891/1933-3196.7.4.225

World Health Organization. (2017). Depression and other common mental disorders. Global Health Estimates. Retrieved from:https://www.who.int/mental_health/ management/depression/prevalence_global_health_ estimates/en/

Disclosure. A.H. is the director of EMDR Institute Germany, which conducts research and teaches in the field of EMDR. He receives income from book publications on EMDR and from training licensed professionals. M.H. receives income from training licensed professionals in EMDR therapy. L.O. receives income as a supervisor of EMDR therapy and as an invited speaker at national and international EMDR conferences. The other authors have no relevant financial interest or affiliations with any commercial interests related to the subjects discussed within this article.

Correspondence regarding this article should be directed to Sara Carletto, Department of Clinical and Biological Sciences, University of Turin, Regione Gonzole 10, 10043 Orbassano (TO), Italy. E-mail: sara.carletto@unito.it 